\title{
COX-2, Inflammatory Secreted PLA2, and Cytoplasmic PLA2 Protein Expression in Small Bowel Adenocarcinomas Compared with Colorectal Adenocarcinomas
}

Dominique Wendum, M.D., Magali Svrcek, M.D., Valérie Rigau, M.D., Pierre-Yves Boëlle, Ph.D., Nicole Sebbagh, Rolland Parc, M.D., Joëlle Masliah, Ph.D., Germain Trugnan, M.D., Ph.D., Jean-François Fléjou, M.D., Ph.D.

Departments of Pathology (DW, MS, VR, NS, J-FF) and Digestive Surgery (RP), Hôpital Saint-Antoine; INSERM U538 (DW, JM, GT) and INSERM U444 (P-YB), Faculté de médecine Saint-Antoine; and INSERM U434 (VR, NS, J-FF), Fondation Jean Dausset-CEPH, Paris, France

Cyclooxygenase-2 (COX-2), human synovial inflammatory secreted phospholipase A2 (sPLA2) and cytoplasmic phospholipase A2 (cPLA2) are involved in eicosanoid production and also seem to participate in colorectal tumorigenesis. As there are no data regarding these enzymes in human small bowel tumors, we wanted to determine whether they were involved in human small bowel tumorigenesis, and whether their expression was different in small bowel compared to colorectal adenocarcinomas, as suggested by animal studies. We studied their protein expression by immunohistochemistry in 25 small bowel adenocarcinomas and compared it to 48 colorectal adenocarcinomas. Seventy-six percent of the small bowel and $88 \%$ of the colorectal adenocarcinomas had a moderate or strong COX-2 expression. Sixty-eight percent of the small bowel and $67 \%$ of the colorectal adenocarcinomas had a moderate or strong sPLA2 expression. Forty-eight percent of the small bowel and $35 \%$ of the colorectal adenocarcinomas had a moderate or strong cPLA2 expression. In conclusion, the increased expression of COX-2, sPLA2, and sometimes cPLA2 in both small bowel and colorectal adenocarcinomas is in accordance with the likely eicosanoid involvement in tumor development. The same pattern of protein expression found in both types of adenocarcinoma contradicts experimental results in mice. Moreover,

Copyright $\odot 2003$ by The United States and Canadian Academy of Pathology, Inc.

VOL. 16, NO. 2, P. 130, 2003 Printed in the U.S.A.

Date of acceptance: October 28, 2002.

Address reprint requests to: Dominique Wendum, M.D., Service d'Anatomie Pathologique, Hôpital Saint-Antoine, 184, rue du faubourg

Saint-Antoine, 75571 Paris Cedex 12, France; fax: 33-01-4928-2878; e-mail: dominique.wendum@sat.ap-hop-paris.fr.

DOI: 10.1097/01.MP.0000052101.58988.1F our results strengthen the similarities between these two types of human cancer.

KEY WORDS: Colorectal cancer, COX-2, Cytoplasmic PLA2, Immunohistochemistry, Inflammatory secreted PLA2, Small bowel cancer.

Mod Pathol 2003;16(2):130-136

Primary adenocarcinomas of the small bowel are rare although the small intestine constitutes almost $90 \%$ of the mucosal surface of the digestive tract. They are more frequent in the duodenum, followed by jejunum and ileum. Familial adenomatous polyposis, hereditary nonpolyposis colorectal cancer (HNPCC) syndrome, Peutz-Jeghers syndrome, Crohn's disease, cystic fibrosis, and cholecystectomy increase the risk of small bowel cancer $(1,2)$. Although 50 times less frequent, small bowel adenocarcinoma share most of the risk factors and the adenoma-carcinoma sequence with colorectal adenocarcinoma (3). Molecular studies showed that some genes (such as K-ras and p53) involved in colorectal adenocarcinoma are altered in the same way and same frequency in small bowel adenocarcinoma (4-6). However, some studies suggested that adenocarcinoma of the small bowel could have a somewhat different genetic pathway compared to colorectal adenocarcinoma, particularly with respect to the apc gene mutation or allelic loss $(4,7$, 8).

Besides these classical genes, several enzymes involved in eicosanoid production also seem to be involved in colorectal tumorigenesis, particularly cyclooxygenase-2 (COX-2) and some phospholipases A2 (inflammatory secreted PLA2 or Type IIA PLA2 and cytoplasmic PLA2 or Type IV PLA2). COX-2, sPLA2, and cPLA2 participate in eicosanoid production, particularly prostaglandin E2 (PGE2), 
which seems involved in intestinal tumor development (9-11).

PLA2 activation releases free arachidonic acid from membrane phospholipids. Then, COX changes free arachidonic acid into prostaglandin $\mathrm{H} 2$ that is further converted into a variety of prostaglandins by different prostaglandin synthases. Several different PLA2 are expressed in human cells, including cytosolic PLA2 (cPLA2) and inflammatory secretory PLA2 (sPLA2) (12). cPLA2 preferentially hydrolyses sn-2 arachidonic acid that is the limiting substrate of COX for prostaglandin production. On the contrary, sPLA2 is not specific for phospholipids, having an arachidonic acid at the sn-2 position but also seeming to be involved in prostaglandin production (13).

In human colorectal tumors, several studies showed that COX-2, sPLA2, and cPLA2 could be involved in tumor development. First, COX-2 is expressed in response to growth factors and cytokines (14), and is overexpressed in most colorectal adenocarcinomas and adenomas (15-17). Treatment with selective COX-2 inhibitors leads to a significant reduction in the number of colorectal polyps in familial adenomatous polyposis (18). Second, sPLA2 is overexpressed in colorectal adenomas in familial adenomatous polyposis (19), although the only study published so far showed no changes in sPLA2 expression in colorectal adenocarcinomas (20). Finally, cPLA2 was shown to be moderately overexpressed in colorectal cancer in two studies $(20,21)$. Whereas COX-2, sPLA2, and cPLA2 expression was studied in human colorectal tumors, it has never been studied in human small bowel tumors. However, these enzymes (COX-2, sPLA2, and cPLA2) have been studied in small bowel tumor development in animal models.

In these models these enzymes seem to be involved in small bowel tumor development but some differences are observed between small bowel and colorectal tumorigenesis. First, in animal models of familial adenomatous polyposis, COX-2 is overexpressed in both colon and small bowel adenomas. Moreover, COX-2 knockout or treatment with selective COX-2 inhibitors results in a reduction or suppression of intestinal polyposis (22-24). Second, in the Min mouse animal model, which develops mainly small bowel adenomas, expression of sPLA2 leads to a resistant phenotype, with development of only a few polyps (25). Finally, cPLA2 was shown to be overexpressed in small bowel adenomas and not in colonic adenomas, and knockout of cPLA2 dramatically modified small bowel polyp development but not colonic polyp development in these models $(26,27)$.

In this study, we wanted to determine whether COX-2, sPLA2, and cPLA2 could be involved in human small bowel tumorigenesis, and whether their expression could be different in small bowel adenocarcinomas compared to colorectal adenocarcinomas, as suggested by animal studies. As the genetic pathway of tumor development, and especially the presence of microsatellite instability, also influences the expression of some of these enzymes $(28,29)$, we studied COX-2, sPLA2, and cPLA2 protein expression in a series of human small bowel adenocarcinomas without microsatellite instability and compared it to a series of human colorectal adenocarcinomas having the same genetic phenotype.

\section{MATERIALS AND METHODS}

\section{Small Bowel Adenocarcinomas}

Twenty-five formalin-fixed, paraffin-embedded tumors were studied. These tumors belonged to an original series of 34 non-familial, non-ampullary primary adenocarcinomas of the small bowel selected from the pathology files of Saint-Antoine, Rothschild and Beaujon Hospitals from 1988 to 2001. All samples were surgical resections. In order to have primary and non-familial cancers, we excluded patients with pre- or coexisting malignancy, and patients having a familial adenomatous polyposis or fulfilling Amsterdam's diagnostic criteria for HNPCC syndrome. In this series of 34 tumors, six tumors fixed with Bouin's fixative were excluded because immunostaining failed in these samples. To have an homogeneous group of tumors according to carcinogenesis, we also excluded three cases with microsatellite instability. Finally, 15 male and 9 female patients with a mean age of 56 years (range, 37-84 y) presented with adenocarcinoma of the duodenum ( $n=11,44 \%)$, jejunum $(n=8,32 \%)$, and ileum ( $n=6,24 \%)$. Four patients had Crohn's disease and one patient had two intestinal tumors. Thirteen tumors were well differentiated (52\%), 6 moderately differentiated $(24 \%)$, and 6 poorly differentiated $(24 \%)$. None of the tumors had a mucinous histology, and one tumor (4\%) had an adenomatous component. Twenty-two tumors (88\%) were Stage T3 or T4 according to the TNM classification (30). Fourteen patients had lymph node metastases $(56 \%)$ and three patients had distant metastasis (12.5\%).

\section{Colorectal Adenocarcinomas}

These 25 small bowel adenocarcinomas were compared with 48 paraffin-embedded colorectal adenocarcinomas without microsatellite instability. These tumors belonged to a series of colorectal cancers selected from the pathology files of SaintAntoine hospital. All samples were surgical resections. To have non-familial cancers, we excluded 
patients having a familial adenomatous polyposis or fulfilling Amsterdam's diagnostic criteria for HNPCC syndrome. Tumors with microsatellite instability were excluded for this study.

Thirty-one male and 17 female patients with a mean age of 65.5 years (range 28-93) presented with adenocarcinoma of the left colon $(n=20$, $41.7 \%)$, right colon ( $n=18,37.5 \%)$, or rectum $(n=$ 10, 20.8\%). Thirty-eight were well differentiated (79.2\%), 7 moderately differentiated (14.6\%) and 3 poorly differentiated (6.2\%). Three tumors had a mucinous histology (6.25\%), and 9 tumors had an adenomatous component (18.75\%). Thirty-seven (77\%) tumors were stage T3 or T4 according to the TNM classification (30). Eighteen patients had lymph node metastases (37.5\%), and 5 patients had distant metastasis (10.4\%).

\section{Microsatellite Instability Assessment}

Microsatellite phenotype was determined by polymerase chain reaction (PCR) at six microsatellite loci and immunohistochemistry. Five loci belonged to the reference panel (BAT25, BAT26, D2S123, D5S346, D17S250) and the sixth locus was an alternative loci (Type II transforming growth factor $\beta$ receptor) according to the National Cancer Institute Workshop on microsatellite instability (31). Cancers showing electrophoretic shifts at two or more loci were considered as having microsatellite instability. We also assessed microsatellite instability by immunohistochemical evaluation of hMLH1 and hMSH2 protein expression (antihuman MLH1 monoclonal antibody, clone G168728, 1/70, BD Pharmigen, and anti-human MSH2 monoclonal antibody, clone FE-11, 1/100, Oncogene Research Products) (32). A loss of hMLH1 or hMSH2 protein expression was observed in all the tumors with microsatellite instability and in none of the tumors without microsatellite instability assessed by PCR.

\section{Immunohistochemistry}

COX-2 and cPLA2 protein expression was studied by immunohistochemistry on the whole series of small bowel and colorectal adenocarcinomas. sPLA2 protein expression was evaluated on the 25 small bowel adenocarcinomas and on 27 colorectal adenocarcinomas.

Consecutive $4-\mu \mathrm{m}$ tissue sections were deparaffinized in xylene and rehydrated in graded alcohol dilutions. Immunolabeling was performed using an avidin-biotin-peroxidase technique (Vectastain ABC Kit, Vector, Burlingame, CA). The following antibodies were used: (1) COX-2 mouse antihuman monoclonal antibody (Cayman Chemical, Ann Arbor, MI) at a dilution of $1 / 500$; (2) cPLA2 mouse monoclonal antibody (sc454, Santa-Cruz Biotechnology, Santa-Cruz, CA) at a dilution of 1/100; (3) sPLA2 (human synovial) rabbit polyclonal antiserum (Cayman Chemical, Ann Arbor, MI) at a dilution of $1 / 200$. Before immunostaining, endogenous peroxidase activity was inhibited with $0.1 \%$ hydrogen peroxide in methanol for 30 minutes. Microwave antigen retrieval was performed for cPLA2 and COX-2 immunostaining $(750 \mathrm{~W}$ during $15 \mathrm{~min}-$ utes, then $150 \mathrm{~W}$ during 15 minutes in citrate buffer (0.01 mol/L, pH 6)). Color development was achieved with 3-amino-9-ethyl-carbazole, and sections were finally counterstained with hematoxylin. Negative controls included omission of the primary antibody and antibody preadsorption with its cognate blocking peptide for cPLA2 and COX-2 or purified protein for sPLA2 (Fig. 1).

Superficial positive interstitial cells were used as internal control for COX-2 staining. Labeled Paneth cells were used as internal control for sPLA2 staining (33). A colon carcinoma overexpressing cPLA2, assessed by Western blot and immunohistochemistry, was used as positive external tissue control for cPLA2 staining (Fig. 2).

\section{Assessment of Stains}

COX-2 immunostaining was evaluated by one author (DW). sPLA2 and cPLA2 immunostainings were independently evaluated by two authors (DW,
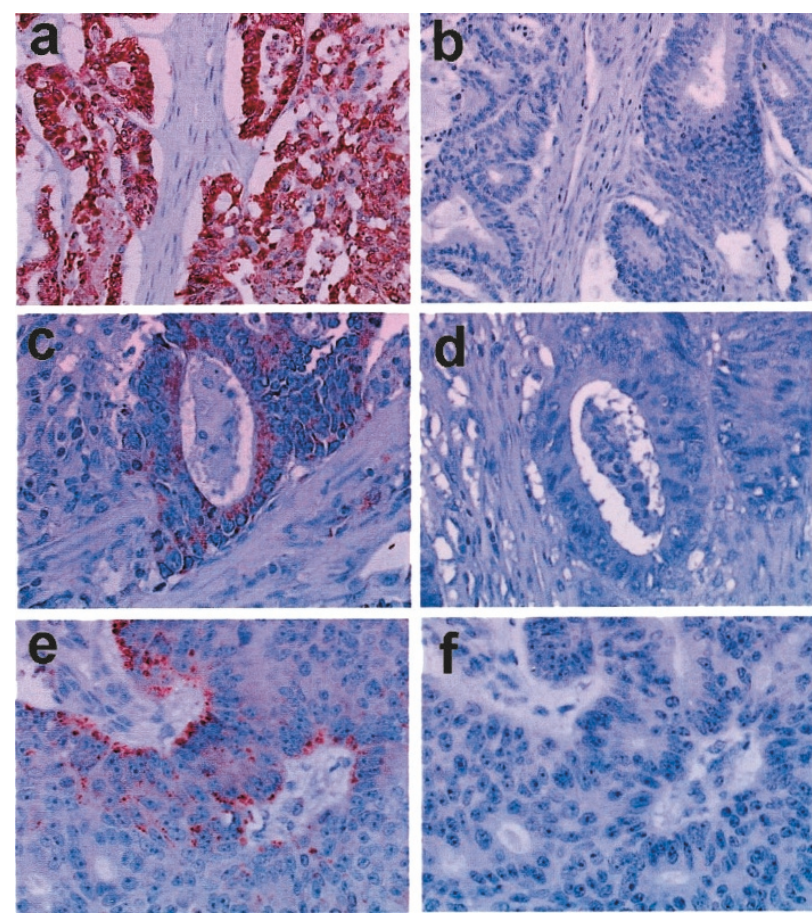

FIGURE 1. Immunostaining controls $(200 \times)$. COX-2 immunostaining (A) without and (B) with antibody preabsorption. sPLA2 immunostaining (C) without and (D) with antibody preabsorption. cPLA2 immunostaining (E) without and (F) with antibody preabsorption. 

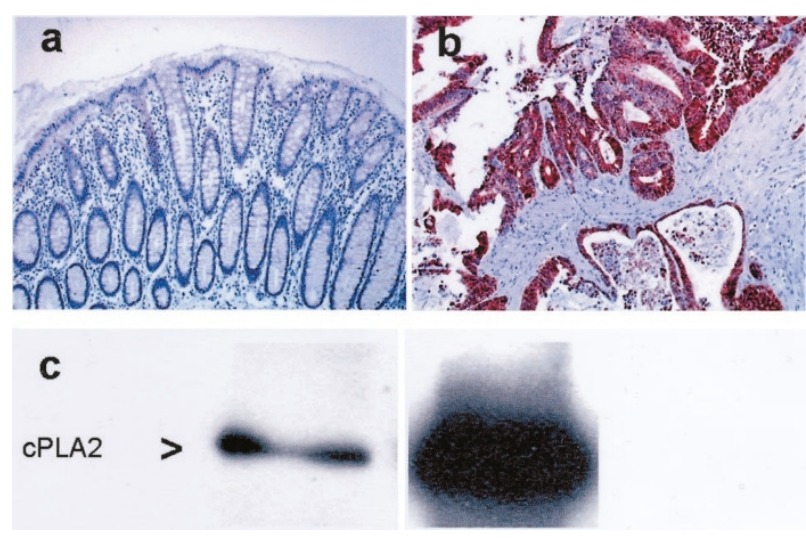

FIGURE 2. CPLA2 positive external control. cPLA2 immunostaining in the colonic normal mucosa (A) and the tumoral tissue (B) in a case chosen as positive external control, and the corresponding Western blot analysis $(\mathbf{C})$.

JFF). Any tumor with a different final assessment was re-evaluated by a consensus review. Inter observer agreement between the two readers was excellent for both sPLA2 (weighted kappa $=0.89$ ) and cPLA2 (weighted kappa $=0.93$ ).

The extent of staining was graded as follows: 0 , staining in $<1 \%$ of tumor cells; 1 , staining in 1 to $20 \%$ of tumor cells; 2 , staining in 20 to $50 \%$ of tumor cells; 3 , staining in $>50 \%$ of tumor cells. The overall intensity of staining was also assessed as follows: 0 , no staining; 1, weak staining; 2, moderate staining; 3 , strong staining. The final score (range from 0 to 9 ) was obtained by multiplying the extent of staining with the intensity. When the final score was 0 , we considered that there was no expression. A final score ranging from 1 to 3 defined a weak expression, a final score of 4 or 6 defined a moderate expression, and a final score of 9 defined a strong expression.

\section{Statistical Analysis}

Interrater agreement for sPLA2 and cPLA2 was estimated by the weighted kappa. Distributions of protein expression levels were compared using the Fisher exact test. All tests were two sided and considered significant if the $P$ value was $<.05$. Comparisons were made using the R-software.

\section{RESULTS}

\section{Small Bowel Adenocarcinomas}

Protein expressions of COX-2, sPLA2, and cPLA2 in the 25 primary small bowel adenocarcinomas are summarized in Table 1 and Figure 4.

\section{COX- 2 Expression in Small Bowel Adenocarcinomas}

See Figure 3, A-B. Most tumors had a moderate or strong COX-2 expression (76\%; Table 1, Fig. 4).
TABLE 1. COX-2, sPLA2, and cPLA2 Protein Expression in the 25 Small Bowel Adenocarcinomas

\begin{tabular}{llrrr}
\hline & Absent & Weak & Moderate & \multicolumn{1}{c}{ Strong } \\
\hline COX-2 & $2(8 \%)$ & $4(16 \%)$ & $6(24 \%)$ & $13(52 \%)$ \\
sPLA2 & $4(16 \%)$ & $4(16 \%)$ & $10(40 \%)$ & $7(28 \%)$ \\
cPLA2 & $7(28 \%)$ & $6(24 \%)$ & $7(28 \%)$ & $5(20 \%)$ \\
\hline
\end{tabular}

COX-2 staining was mostly located in tumor cells and was always cytoplasmic (Fig. 3A). Intense positive superficial interstitial cells were always present, even in tumors with no COX-2 expression in cancerous cells and were therefore used as internal positive control.

In the adjacent normal mucosa, there was often a weak or moderate COX-2 staining (Fig. 3B). In the four cases associated with Crohn's disease the adjacent mucosa had a moderate COX-2 expression.

\section{sPLA2 Expression in Small Bowel \\ Adenocarcinomas}

See Figure 3, C-D. Most tumors had a moderate or strong sPLA2 expression (68\%; Table 1, Fig. 4). sPLA2 staining was always located in the tumor cells and was cytoplasmic and granular (Fig. 3C).
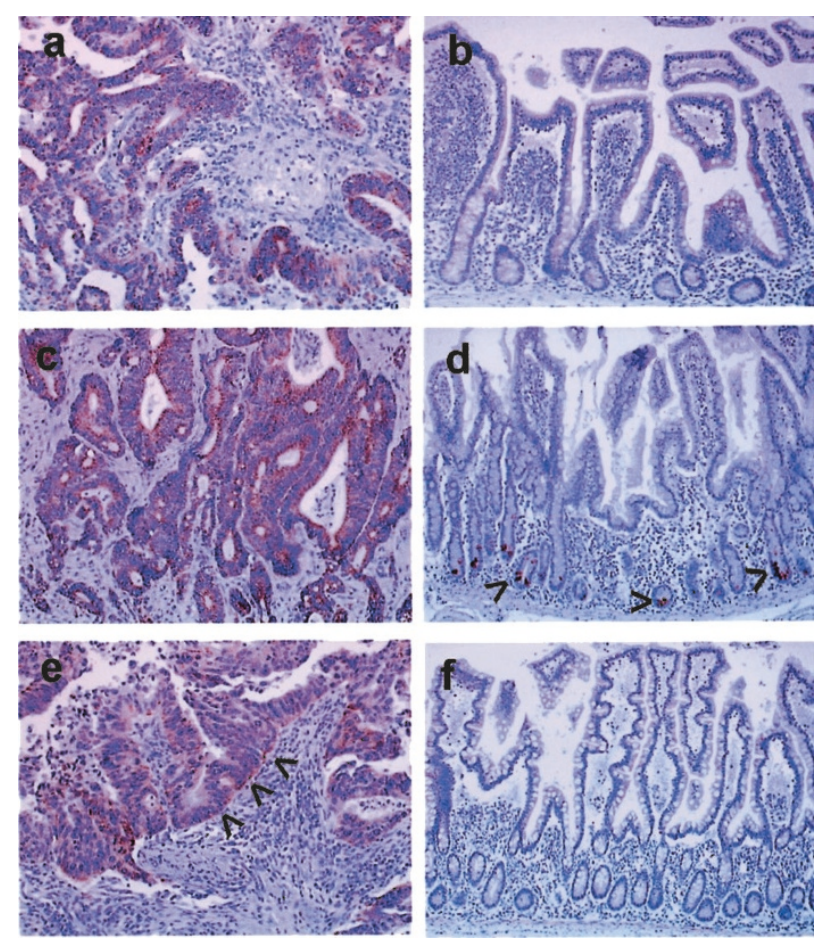

FIGURE 3. COX-2, sPLA2, and cPLA2 protein expression in small bowel adenocarcinomas and in the adjacent normal mucosae $(100 \times)$. COX-2: A, strong immunostaining in a small bowel adenocarcinoma and (B) weak to moderate immunostaining in the adjacent normal epithelial cells. sPLA2: C, strong immunostaining in a small bowel adenocarcinoma and (D) labeled Paneth cells (arrows) in the adjacent normal mucosa. cPLA2: E, strong immunostaining in a small bowel adenocarcinoma with a basal reinforcement (arrows) and (F) weak immunostaining in the adjacent normal epithelium cells and muscularis mucosae. 

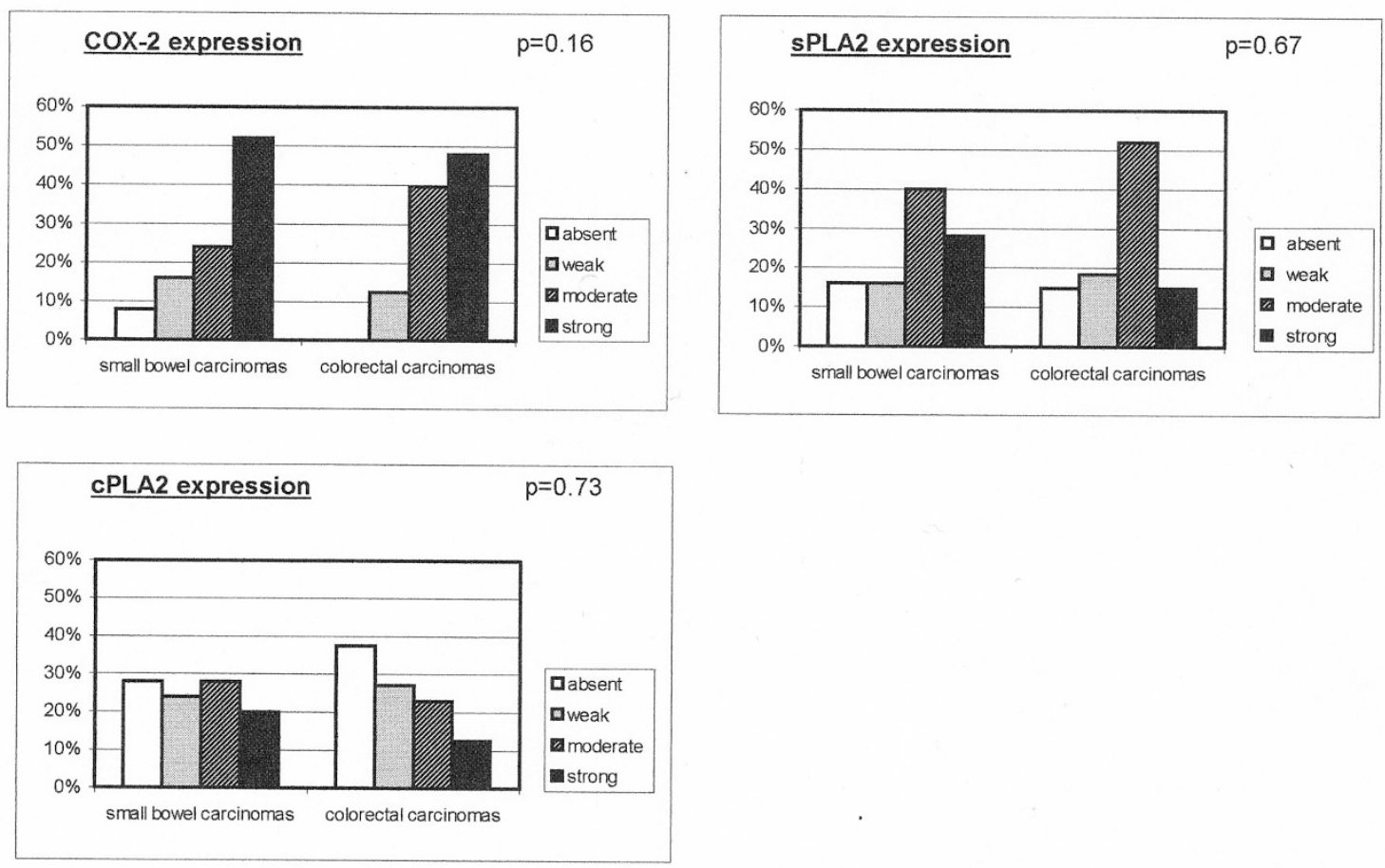

FIGURE 4. Distribution of COX-2, sPLA2, and cPLA2 protein expression in the small bowel and colorectal adenocarcinomas. The statistical analysis compared the protein expression between the small bowel and colorectal adenocarcinomas.

The stroma was always negative even when the tumor was ulcerated.

A strong sPLA2 staining was observed in the Paneth cells and in the lamina elastica of arteries in the normal tissue as previously described (Fig. 3d) (33). There was no or only a very weak sPLA2 expression in the epithelial cells of the normal adjacent mucosa. In the four cases associated with Crohn's disease the adjacent mucosae had a moderate sPLA2 expression.

\section{cPLA2 Expression in Small Bowel Adenocarcinomas}

See Figure 3, E-F. A moderate or strong cPLA2 expression in tumors was observed in $48 \%$ of the cases (Table 1, Fig. 4). In tumors, cPLA2 staining was observed in tumor cells and in rare superficial interstitial cells. These superficial interstitial cells were often located within an ulcerated area. When present, cPLA2 staining in tumor cells was cytoplasmic and granular with a basal intensification (Figs. 1E and 3E).

TABLE 2. COX-2, sPLA2, and cPLA2 Protein Expression in the Colorectal Adenocarcinomas

\begin{tabular}{lcrrr}
\hline & Absent & \multicolumn{1}{c}{ Weak } & Moderate & \multicolumn{1}{c}{ Strong } \\
\hline COX-2 & $0(0 \%)$ & $6(12.5 \%)$ & $19(39.6 \%)$ & $23(47.9 \%)$ \\
sPLA2 & $4(14.8 \%)$ & $5(18.5 \%)$ & $14(51.9 \%)$ & $4(14.8 \%)$ \\
cPLA2 & $18(37.5 \%)$ & $13(27.1 \%)$ & $11(22.9 \%)$ & $6(12.5 \%)$ \\
\hline
\end{tabular}

COX-2 and cPLA2 protein expression was evaluated on the 48 samples. sPLA2 protein expression was evaluated on 27 samples.
cPLA2 staining was observed in the smooth muscle of the muscularis propria and the muscularis mucosae and in rare endothelial cells. There often was a weak staining of normal epithelial cells (Fig. 3F), which was also observed in the adjacent mucosa of the four cases associated with Crohn's disease.

In the small bowel adenocarcinomas, there was neither association between COX-2 and cPLA2 expression $(P=.22)$, nor between COX-2 and sPLA2 expression $(P=.62)$ nor between cPLA2 and sPLA2 expression $(P=.23)$.

\section{Colorectal Adenocarcinomas}

Protein expression of COX-2, sPLA2, and cPLA2 in colorectal adenocarcinomas are summarized in Table 2 and Figure 4.

Immunostainings had the same pattern as in the small bowel adenocarcinomas.

Most colorectal adenocarcinomas had a moderate or strong COX-2 expression $(87.5 \%)$, a moderate or strong sPLA2 expression (66.6\%). A moderate or strong cPLA2 expression was seen in only $35.4 \%$ of the colorectal adenocarcinomas (Table 2, Fig. 4). In the colorectal adenocarcinomas, there was neither association between COX-2 and cPLA2 expression $(P=.99)$, nor between COX-2 and sPLA2 expression $(P=.44)$ nor between cPLA2 and sPLA2 expression $(P=.19)$ 
Comparison between Small

Bowel Adenocarcinomas and

Colorectal Adenocarcinomas

There was no significant difference in COX-2 $(P=.157)$, sPLA2 $(P=.67)$, and cPLA2 $(P=.73)$ protein expression between small bowel and colorectal adenocarcinomas (Fig. 4).

\section{DISCUSSION}

This is the first study that focused on COX-2, sPLA2, and CPLA2 expression in human primary small bowel adenocarcinomas. This retrospective series is relatively large considering the very low incidence of this disease. This series also has the main characteristics usually described in this type of cancer: the frequent duodenum involvement, the presence of Crohn's disease in some cases, a higher incidence in the fifth and sixth decade, and a frequent advanced clinical stage at the time of diagnosis (1).

The increased expression of COX-2, sPLA2, and sometimes cPLA2 that we observed both in small bowel and colorectal adenocarcinomas in our study is in accordance with the likely PGE2 involvement in tumor development since a co-ordinate activation of these enzymes seems necessary to produce prostaglandins $(34,35)$. However, an increased protein expression does not necessarily indicate an important activation or increased function. Therefore further investigations with functional studies on tumor cells are necessary.

We observed a moderate or strong COX-2 expression in most small bowel adenocarcinomas as in colorectal adenocarcinomas. Numerous animal studies and some human studies suggest that selective COX-2 inhibitors could be, as classical NSAID, effective in preventing colorectal tumor development $(18,36,37)$. Our results in small bowel adenocarcinomas suggest that selective COX-2 inhibitors could be possible candidates for preventing this type of tumor.

Small bowel adenocarcinomas resemble colorectal adenocarcinoma with regard to risk factors and the adenoma-carcinoma sequence $(1,3)$. In our series of small bowel adenocarcinomas, we found almost the same pattern of COX-2, sPLA2, and cPLA2 expression as in the series of colorectal adenocarcinomas that we studied concomitantly. Thus, although adenocarcinomas of the small intestine could have a somewhat different genetic pathway compared with colorectal adenocarcinomas (8), we showed that they are alike with regard to COX-2, sPLA2, and cPLA2 protein expression.

Our results are somewhat different from experimental results in mice. Indeed, two studies suggested that cPLA2 expression is involved in small bowel tumor development but not in colonic tumor development $(26,27)$. In our study, we observed cPLA2 overexpression in $48 \%$ of the small bowel adenocarcinomas and in only $35 \%$ of the colorectal adenocarcinomas. Although cPLA2 over expression could preferentially mediate small bowel tumor development, this difference in cPLA2 expression we observed was not significant $(P=.73)$. Regarding sPLA2, this discrepancy between animal model results and human disease is also observed in our study. Indeed, in the Min mouse model of familial adenomatous polyposis, mutations in the sPLA2 gene with a low sPLA2 expression correlate with an increased tumor number suggesting a protective effect of sPLA2 expression on small bowel and colorectal tumor development (25, 38). In contrast, in human familial adenomatous polyposis, sPLA2 expression does not appear to modify the severity of the disease $(39,40)$. Moreover, an increased sPLA2 expression was found in colorectal adenomas of familial adenomatous polyposis patients (19). In accordance with this last result, we also found a strong sPLA2 expression in most small bowel and colorectal adenocarcinomas. Moreover, in the four cases of small bowel adenocarcinomas associated with Crohn's disease the adjacent mucosa had a moderate sPLA2 expression, whereas in the other cases the adjacent mucosa had no or only a very weak sPLA2 expression. This result is also in accordance with previous results (41).

In conclusion, our study showed that human primary adenocarcinomas without microsatellite instability have the same pattern of COX-2, inflammatory secreted PLA2 and cytoplasmic PLA2 protein expression as colorectal adenocarcinomas: COX-2 is overexpressed in most tumors, sPLA2 is frequently overexpressed and cPLA2 is sometimes overexpressed. In contrast with experimental results in mice, cPLA2 overexpression does not seem to be preferentially observed in small bowel tumors in humans. These results suggest that eicosanoid production could be involved in small bowel tumor development in the same way as in colorectal tumor development. However, an increased protein expression does not necessarily indicate an important activation or increased function. Therefore, further investigations with functional studies on tumor cells are necessary.

\section{REFERENCES}

1. Neugut AI, Jacobson JS, Suh S, Mukherjee R, Arber N. The epidemiology of cancer of the small bowel. Cancer Epidemiol Biomarkers Prev 1998;7:243-51.

2. Johnson AM, Harman PK, Hanks JB. Primary small bowel malignancies. Am Surg 1985;51:31-6.

3. Sellner F. Investigations on the significance of the adenomacarcinoma sequence in the small bowel. Cancer 1990;66:702-15.

4. Rashid A, Hamilton SR. Genetic alterations in sporadic and Crohn's-associated adenocarcinomas of the small intestine. Gastroenterology 1997;113:127-35. 
5. Arber N, Neugut AI, Weinstein IB, Holt P. Molecular genetics of small bowel cancer. Cancer Epidemiol Biomarkers Prev 1997;6:745-8.

6. Arber N, Hibshoosh H, Yasui W, Neugut AI, Hibshoosh A, Yao Y, et al. Abnormalities in the expression of cell cyclerelated proteins in tumors of the small bowel. Cancer Epidemiol Biomarkers Prev 1999;8:1101-5.

7. Arai M, Shimizu S, Imai Y, Nakatsuru Y, Oda H, Oohara T, et al. Mutations of the Ki-ras, p53 and APC genes in adenocarcinomas of the human small intestine. Int J Cancer 1997;70:390-5.

8. Wheeler JM, Warren BF, Mortensen NJ, Kim HC, Biddolph $\mathrm{SC}$, Elia $\mathrm{G}$, et al. An insight into the genetic pathway of adenocarcinoma of the small intestine. Gut 2002;50:218-23.

9. Pugh S, Thomas GA. Patients with adenomatous polyps and carcinomas have increased colonic mucosal prostaglandin $\mathrm{E}_{2}$. Gut 1994;35:675-8.

10. Watanabe K, Kawamori T, Nakatsugi S, Ohta T, Ohuchida S, Yamamoto $\mathrm{H}$, et al. Role of the prostaglandin E receptor subtype EP1 in colon carcinogenesis. Cancer Res 1999;59:5093-6.

11. Sonoshita M, Takaku K, Sasaki N, Sugimoto Y, Ushikubi F, Narumiya S, et al. Acceleration of intestinal polyposis through prostaglandin receptor EP2 in Apc(Delta 716) knockout mice. Nat Med 2001;7:1048-51.

12. Six DA, Dennis EA. The expanding superfamily of phospholipase A(2) enzymes: classification and characterization. Biochim Biophys Acta 2000;1488:1-19.

13. Balsinde J, Balboa MA, Insel PA, Dennis EA. Regulation and inhibition of phospholipase A2. Annu Rev Pharmacol Toxicol 1999;39:175-89.

14. Dubois RN, Abramson SB, Crofford L, Gupta RA, Simon LS, Van De Putte LB, et al. Cyclooxygenase in biology and disease. FASEB J 1998;12:1063-73.

15. Eberhart CE, Coffey RJ, Radhika A, Giardiello FM, Ferrenbach S, DuBois RN. Up-regulation of cyclooxygenase 2 gene expression in human colorectal adenomas and adenocarcinomas. Gastroenterology 1994;107:1183-8.

16. Sano H, Kawahito Y, Wilder RL, Hashiramoto A, Mukai S, Asai $\mathrm{K}$, et al. Expression of cyclooxygenase-1 and -2 in human colorectal cancer. Cancer Res 1995;55:3785-9.

17. Hao X, Bishop AE, Wallace M, Wang H, Willcocks TC, Maclouf $\mathrm{J}$, et al. Early expression of cyclo-oxygenase-2 during sporadic colorectal carcinogenesis. J Pathol 1999;187:295-301.

18. Steinbach G, Lynch PM, Phillips RK, Wallace MH, Hawk E, Gordon GB, et al. The effect of celecoxib, a cyclooxygenase-2 inhibitor, in familial adenomatous polyposis. N Engl J Med 2000;342:1946-52.

19. Kennedy BP, Soravia C, Moffat J, Xia L, Hiruki T, Collins S, et al. Overexpression of the nonpancreatic secretory group II PLA2 messenger RNA and protein in colorectal adenomas from familial adenomatous polyposis patients. Cancer Res 1998;58:500-3.

20. Dimberg J, Samuelsson A, Hugander A, Soderkvist P. Gene expression of cyclooxygenase-2, group II and cytosolic phospholipase A2 in human colorectal cancer. Anticancer Res 1998;18:3283-7.

21. Soydan AS, Tavares IA, Weech PK, Temblay NM, Bennett A. High molecular weight phospholipase A2 and fatty acids in human colon tumours and associated normal tissue. Eur J Cancer 1996;32A:1781-7.

22. Oshima M, Dinchuk JE, Kargman SL, Oshima H, Hancock B, Kwong E, et al. Suppression of intestinal polyposis in Apc delta716 knockout mice by inhibition of cyclooxygenase 2 (COX-2). Cell 1996;87:803-9.

23. Oshima M, Murai N, Kargman S, Arguello M, Luk P, Kwong E, et al. Chemoprevention of intestinal polyposis in the Apcdelta716 mouse by rofecoxib, a specific cyclooxygenase- 2 inhibitor. Cancer Res 2001;61:1733-40.

24. Jacoby RF, Seibert K, Cole CE, Kelloff G, Lubet RA. The cyclooxygenase-2 inhibitor celecoxib is a potent preventive and therapeutic agent in the min mouse model of adenomatous polyposis. Cancer Res 2000;60:5040-4.

25. MacPhee M, Chepenik KP, Liddell RA, Nelson KK, Siracusa LD, Buchberg AM. The secretory phospholipase A2 gene is a candidate for the Mom1 locus, a major modifier of ApcMininduced intestinal neoplasia. Cell 1995;81:957-66.

26. Takaku K, Sonoshita M, Sasaki N, Uozumi N, Doi Y, Shimizu T, et al. Suppression of intestinal polyposis in Apc(delta 716) knockout mice by an additional mutation in the cytosolic phospholipase A(2) gene. J Biol Chem 2000;275:34013-6.

27. Hong KH, Bonventre JC, O'Leary E, Bonventre JV, Lander ES. Deletion of cytosolic phospholipase A(2) suppresses Apc(Min)-induced tumorigenesis. Proc Natl Acad Sci U S A 2001;98:3935-9.

28. Sinicrope FA, Lemoine M, Xi L, Lynch PM, Cleary KR, Shen $\mathrm{Y}$, et al. Reduced expression of cyclooxygenase 2 proteins in hereditary nonpolyposis colorectal cancers relative to sporadic cancers. Gastroenterology 1999;117:350-8.

29. Karnes WE Jr, Shattuck-Brandt R, Burgart LJ, DuBois RN, Tester DJ, Cunningham JM, et al. Reduced COX-2 protein in colorectal cancer with defective mismatch repair. Cancer Res 1998;58:5473-7.

30. Sobin LH, Wittekind C, eds. TNM classification of malignant tumors. 5th ed. New York: Wiley-Liss, 1997.

31. Boland CR, Thibodeau SN, Hamilton SR, Sidransky D, Eshleman JR, Burt RW, et al. A National Cancer Institute Workshop on Microsatellite Instability for cancer detection and familial predisposition: development of international criteria for the determination of microsatellite instability in colorectal cancer. Cancer Res 1998;58:5248-57.

32. Lindor NM, Burgart LJ, Leontovich O, Goldberg RM, Cunningham JM, Sargent DJ, et al. Immunohistochemistry versus microsatellite instability testing in phenotyping colorectal tumors. J Clin Oncol 2002;20:1043-8.

33. Nevalainen TJ, Gronroos JM, Kallajoki M. Expression of group II phospholipase A2 in the human gastrointestinal tract. Lab Invest 1995;72:201-8.

34. Murakami M, Shimbara S, Kambe T, Kuwata H, Winstead MV, Tischfield JA, et al. The functions of five distinct mammalian phospholipase A2S in regulating arachidonic acid release. Type IIa and type V secretory phospholipase A2S are functionally redundant and act in concert with cytosolic phospholipase A2. J Biol Chem 1998;273:14411-23.

35. Balsinde J, Balboa MA, Dennis EA. Functional coupling between secretory phospholipase A2 and cyclooxygenase-2 and its regulation by cytosolic group IV phospholipase A2. Proc Natl Acad Sci U S A 1998;95:7951-6.

36. Sheng H, Shao J, Kirkland SC, Isakson P, Coffey RJ, Morrow J, et al. Inhibition of human colon cancer cell growth by selective inhibition of cyclooxygenase-2. J Clin Invest 1997;99:2254-9.

37. Reddy BS, Hirose Y, Lubet R, Steele V, Kelloff G, Paulson S, et al. Chemoprevention of colon cancer by specific cyclooxygenase-2 inhibitor, celecoxib, administered during different stages of carcinogenesis. Cancer Res 2000;60:293-7.

38. Cormier RT, Hong KH, Halberg RB, Hawkins TL, Richardson P, Mulherkar R, et al. Secretory phospholipase Pla2g2a confers resistance to intestinal tumorigenesis. Nat Genet 1997; 17:88-91.

39. Dobbie Z, Muller H, Scott RJ. Secretory phospholipase A2 does not appear to be associated with phenotypic variation in familial adenomatous polyposis. Hum Genet 1996;98:386-90.

40. Spirio LN, Kutchera W, Winstead MV, Pearson B, Kaplan C, Robertson M, et al. Three secretory phospholipase A(2) genes that map to human chromosome 1P35-36 are not mutated in individuals with attenuated adenomatous polyposis coli. Cancer Res 1996;56:955-8.

41. Haapamaki MM, Gronroos JM, Nurmi H, Alanen K, Nevalainen TJ. Gene expression of group II phospholipase A2 in intestine in Crohn's disease. Am J Gastroenterol 1999;94:713-20. 